\title{
68. Sur les équations fondamentales dans la géométrie conforme des sous-espaces.
}

\author{
Par Kentaro Yano. \\ Institut Mathématique, Université Impériale de Tokyo. \\ (Comm. by S. KAKEYA, M.I.A., July 12, 1943.)
}

$\S 0$. Dans quelques travaux antérieurs' ${ }^{1)}$, nous avons trouvé les équations de Gauss, de Codazzi et de Ricci dans la géométrie conforme des sous-espaces riemanniens.

Mais, si l'on étudie la condition nécessaire et suffisante pour que les trois tenseurs conformes fondamentaux $\rho^{2} g_{j k}, \rho M_{j k P}$ et $L_{P Q k}$ déterminent un sous-espace plongé dans un espace euclidien, on obtient cinq relations entre ces tenseurs conformes fondamentaux, dont les trois sont les équations conformes de Gauss, de Codazzi et de Ricci pour un sous-espace dans un espace euclidien".

Le but de cette Note est de trouver les deux autres équations conformes pour un sous-espace dans un espace riemannien général.

Pour cela, on introduit un tenseur conforme $C_{j k}$ et une scalaire conforme $C$ qui joueront un rôle très important dans la théorie des espaces à connexion conforme et la géométrie conforme des sous-espaces riemanniens.

Une autre application de ces quantités conformes sera trouvée dans la Note suivante.

$\S 1$. Considérons un espace à connexion conforme normale $C_{n}{ }^{3)}$, et prenons, dans chaque espace tangent de Möbius $M_{n}$, le repère de Veblen $\left[A_{0}, A_{\lambda}, A_{\infty}\right]^{4}$, alors, la connexion conforme normale sera représentée par les formules de la forme

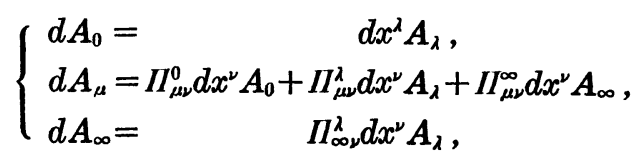

où

1) K. Yano: Sur les équations de Gauss dans la géométrie conforme des espaces de Riemann, Proc. 15 (1939), 247-252; Sur les équations de Codazzi dans la géométrie conforme des espaces de Riemann, Proc. 15 (1939), 340-344; K. Yano et Y. Mutô: Sur la théorie des espaces à connexion conforme normale et la géométrie conforme des espaces de Riemann, Journal of the Faculty of Science, Imperial University of Tokyo, 4 (1941), 117-169.

2) K. Yano et Y. Mutô : Sur le théorème fondamental dans la géométrie conforme des sous-espaces riemanniens, Proceedings of the Physico-Math. Soc. Japan, 24 (1942), 437-449.

3) Voir, K. Yano: Sur la théorie des espaces à connexion conforme, Journal of the Faculty of Science, Imperial University of Tokyo, 4 (1939), 1-59, et $K$. Yano et Y. Mutô: Sur la théorie des espaces à connexion conforme normale et la géométrie conforme des espaces de Riemann, déjà- cité.

4) Les indices $\left\{\begin{array}{l}\lambda, \mu, \nu, \ldots \\ i, j, k, \ldots \text { parcourent les symboles }\left\{\begin{array}{l}1,2, \ldots \ldots, n \\ P, Q, R, \ldots\end{array}\right. \\ \dot{i}, \dot{2}, \ldots \ldots, \dot{m} \\ \dot{m}, \dot{m}+\dot{i}, \ldots, \dot{n}\end{array}\right.$ 
$A_{0} A_{0}=0, A_{\mu} A_{\nu}=g_{\mu \nu}, A_{\infty} A_{\infty}=0, A_{0} A_{\lambda}=0, A_{\infty} A_{\lambda}=0, A_{0} A_{\infty}=-1$,

et $\quad \Pi_{\mu \nu}^{0}=-\frac{R_{\mu \nu}}{n-2}+\frac{R g_{\mu \nu}}{2(n-1)(n-2)}, \quad \Pi_{\infty \nu}^{\lambda}=g^{\lambda \mu} I_{\mu \nu}^{0}, \quad \Pi_{\mu \nu}^{\infty}=g_{\mu \nu}$,

$$
I_{\mu \nu}^{\lambda}=\left\{\begin{array}{c}
\lambda \\
\mu \nu
\end{array}\right\}=\frac{1}{2} g^{\lambda a}\left(\frac{\partial g_{a \mu}}{\partial x^{\nu}}+\frac{\partial g_{a \nu}}{\partial x^{\mu}}-\frac{\partial g_{\mu \nu}}{\partial x^{\alpha}}\right),
$$

$R_{\mu \nu}$ et $R$ étant respectivement le tenseur de Ricci et la courbure scalaire formés avec les composantes du tenseur de courbure

$$
R_{\cdot \mu \nu \omega}^{\lambda}=\frac{\partial\left\{\begin{array}{c}
\lambda \\
\mu \nu
\end{array}\right\}}{\partial x^{\omega}}-\frac{\partial\left\{\begin{array}{c}
\lambda \\
\mu \omega
\end{array}\right\}}{\partial x^{\nu}}+\left\{\begin{array}{c}
a \\
\mu \nu
\end{array}\right\}\left\{\begin{array}{c}
\lambda \\
a \omega
\end{array}\right\}-\left\{\begin{array}{c}
a \\
\mu \omega
\end{array}\right\}\left\{\begin{array}{c}
\lambda \\
a \nu
\end{array}\right\} .
$$

Cela étant, considérons un sous-espace à $m$ dimensions défini par les équations paramétriques

$$
x^{\lambda}=x^{\lambda}\left(x^{\dot{i}}, x^{\dot{2}}, \ldots, x^{\dot{m}}\right),
$$

et définissons, à chaque point de ce sous-espace, le repère $\left[A_{\dot{0}}, A_{i}\right.$, $\left.A_{P}, A_{\infty}\right]$ par

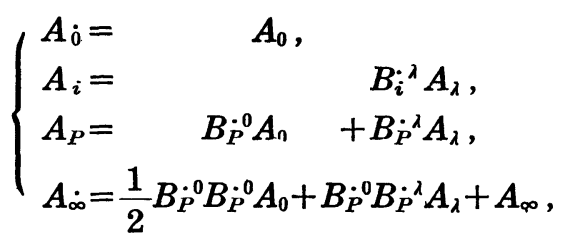

où

$$
B_{i}^{\cdot \lambda}=\frac{\partial x^{\lambda}}{\partial x^{i}}, \quad g_{j k}=g_{\mu \nu} B_{i}^{\mu \mu} B_{k^{\nu}}^{\nu},
$$

et $B_{\dot{P}}^{\lambda}$ sont définis par

$$
g_{\mu \nu} B_{j}^{\mu \mu} B_{\dot{P}}^{\nu}=0, \quad g_{\mu \nu} B_{\dot{P}}^{\mu} B_{\dot{Q}^{\nu}}=\delta_{P Q} .
$$

Or, si l'on pose la condition que la moyenne de $d A_{\dot{0}} d A_{P}$ par rapport à $d x^{i}$ s'annule, soit, qu'on ait

on a

$$
\left(B_{\dot{P}}^{0} g_{j k}-g_{a \beta} H_{j k}^{* a} B_{\dot{P}}^{\beta}\right) g^{j k}=0,
$$

$$
B_{\dot{P}}^{0}={ }_{m}^{1} H_{\cdot a P}^{a},
$$

ou nous avons posé

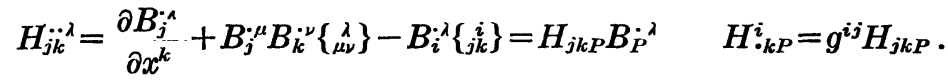

Les points et les sphères $\left[A_{\dot{0}}, A_{i}, A_{P}, A_{\dot{\infty}}\right]$ étant ainsi définis, leurs déplacements d'après la connexion conforme de l'espace ambiant sont donnes par

$$
\left\{\begin{array}{l}
d A_{\dot{0}}=\quad d x^{2} A_{i}, \\
d A_{j}=I I_{j k}^{0} d x^{k} A_{0}+I I_{j k}^{i} d x^{k} A_{i}+I_{j k P} d x^{k} A_{P}+I_{j k}^{\dot{\infty}} d x^{k} A_{\dot{\infty}}, \\
d A_{P}=I I_{P k}^{\dot{j}} d x^{k} A_{\dot{0}}+I_{P k}^{i} d x^{k} A_{i}+I I_{P Q k} d x^{k} A_{Q}, \\
d A_{\dot{\infty}}=\quad I_{\dot{\infty} k}^{i} d x^{k} A_{i}+\Pi_{\infty P k} d x^{k} A_{P},
\end{array}\right.
$$


où

et

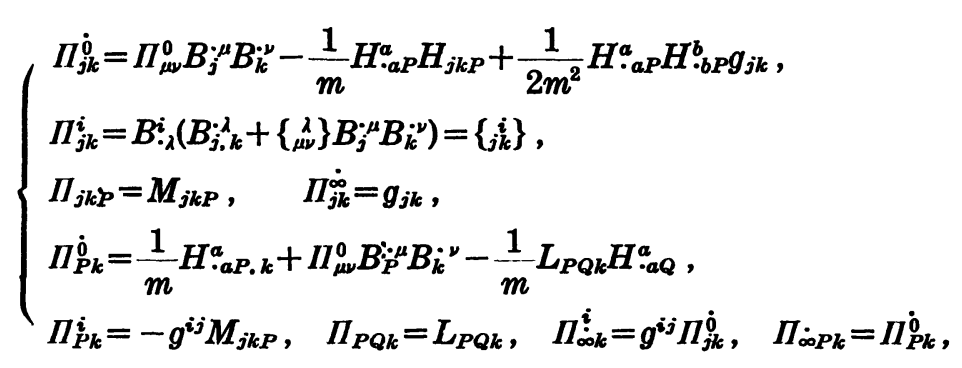

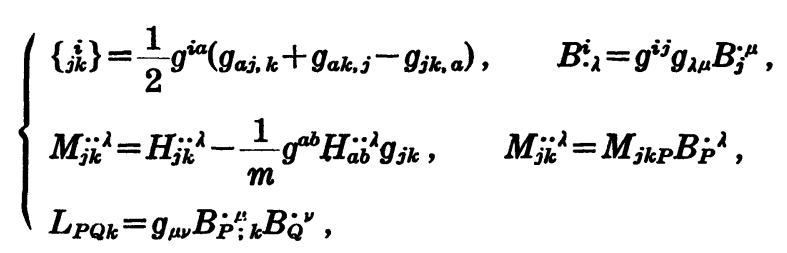

la virgule et le point-virgule désignant respectivement la dérivée ordinaire et la dérivée covariante.

Cela étant, la connexion conforme induite sur le sous-espace (1.2) est définie par

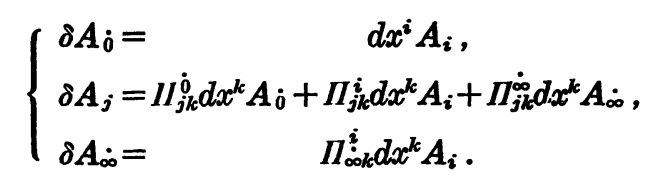

Mais, on peut, d'autre part, donner aussi la connexion conforme normale intrinsèque au sous-espace (1.2) par

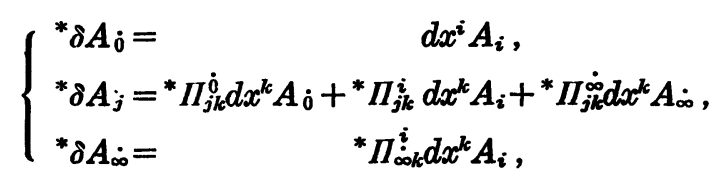

où

$$
\begin{aligned}
& { }^{*} I_{j k}^{\dot{0}}=-\frac{R_{j k}}{m-2}+\frac{g^{a b} R_{a b} g_{j k}}{2(m-1)(m-2)}, \quad{ }^{*} I_{j k}^{i}=\left\{\begin{array}{c}
i \\
j k
\end{array}\right\}, \\
& { }^{*} I_{j k}^{\dot{\infty}}=g_{j k}, \quad{ }^{*} \Pi_{\dot{c} k}^{i}=g^{i j *} \Pi_{j k}^{\dot{0}},
\end{aligned}
$$

$R_{j k}$ étant le tenseur de Ricci formés avec les composantes du tenseur de courbure

$$
R_{\cdot j k h}^{i}=\left\{\begin{array}{c}
i \\
j k
\end{array}\right\}_{, h}-\left\{\begin{array}{c}
i \\
j h
\end{array}\right\}_{, k}+\left\{\begin{array}{c}
a \\
j k
\end{array}\right\}\left\{\begin{array}{c}
i \\
a h
\end{array}\right\}-\left\{\begin{array}{c}
a \\
j h
\end{array}\right\}\left\{\begin{array}{c}
i \\
a k
\end{array}\right\}
$$

§2. On voit, d'après ce qui est dit dans le paragraphe précédent, que la condition nécessaire et suffisante pour que la connexion induite et la connexion intrinsèque sur le sous-espace coïncident est que le tenseur $C_{j k}={ }^{*} I_{j k}^{\dot{0}}-\Pi_{j k}^{\dot{0}}$ s'annule. Dans ce qui suit, on va calculer ce tenseur. 
No. 7.] Sur les équations fondamentales dans la géométrie conforme.

Le tenseur $\Pi_{j k}^{\dot{0}}$ est donné par (1.6), soit, par

$$
\Pi_{j k}^{\dot{0}}=\Pi_{\mu \nu}^{0} B_{j}^{\cdot \mu} B_{k}^{\nu}-\frac{1}{m} H_{\cdot a \lambda}^{a} H_{j k}^{\alpha}+\frac{1}{2 m^{2}} H_{\cdot a \lambda}^{a} H_{\cdot b}^{b \cdot \lambda} g_{j k} \text {. }
$$

Pour calculer le tenseur ${ }^{*} \Pi_{j k \text {, }}^{\dot{j}}$ prenons d'abord les équations de Gauss pour le sous-espace

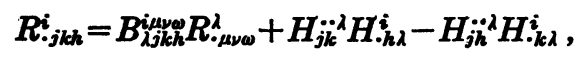

où et dans la suite on pose, pour simplicité,

$B_{\lambda j k h}^{i \mu \nu \omega}=B_{. j}^{i} B_{j}^{\cdot \mu} B_{k}^{\cdot \nu} B_{h}^{\cdot \omega} ; \quad B_{j k}^{\mu \nu}=B_{j}^{\cdot \mu} B_{k}^{\cdot \nu}, \quad B^{\mu \nu}=B_{j k}^{\mu \nu} g^{j k}, \quad B_{\lambda}^{\omega}=B_{\cdot \lambda}^{i} B_{i}^{\cdot \omega}$.

Or, en contractant $B_{j j k n}^{i \mu \nu}$ au tenseur conforme de courbure de Weyl

on trouve

$$
C_{. \mu \nu \omega}^{\lambda}=R_{. \mu \nu \omega}^{\lambda}+I I_{\mu \nu}^{0} \delta_{\omega}^{\lambda}-\Pi_{\mu \omega \omega}^{0} \delta_{\nu}^{\lambda}+g_{\mu \nu} I_{\infty \omega \omega}^{\lambda}-g_{\mu \omega} \Pi_{\infty \nu}^{\lambda},
$$

$$
\begin{aligned}
& B_{j j k h}^{i \mu \nu \omega} C_{. \mu \nu \omega}^{\lambda}=B_{j j k h}^{i \mu \nu \omega} R_{. \mu \nu \omega}^{\lambda}+\Pi_{\mu \nu}^{0} B_{j}^{\mu \mu} B_{k}^{\cdot \nu} \partial_{h}^{i}-\Pi_{\mu \nu}^{0} B_{j}^{-\mu} B_{h}^{\cdot \nu} \delta_{k}^{i} \\
& +g_{j k} \Pi_{\mu \nu}^{0} B_{a}^{\cdot \mu} B_{h}^{\cdot \nu} g^{a i}-g_{j h} \Pi_{\mu \nu}^{0} B_{a}^{\cdot \mu} B_{k}^{\cdot \nu} g^{a i}
\end{aligned}
$$

En substituant (2.2) dans cette équation, on obtient

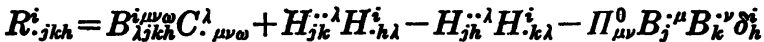

$$
\begin{aligned}
& +\Pi_{\mu \nu}^{\dagger} B_{j}^{\cdot \mu} B_{h}^{\cdot \nu} \delta_{k}^{i}-g_{j k} \Pi_{\mu \nu}^{0} B_{a}^{\cdot \mu} B_{k}^{\cdot \nu} g^{\alpha i}+g_{j h} \Pi_{\mu \nu}^{0} B_{a}^{\cdot \mu} B_{k}^{\cdot \nu} g^{a i},
\end{aligned}
$$

d'où, en contractant par rapport à $i$ et $h$, on trouve

$$
R_{j k}=B_{\lambda}^{\bullet} B_{j k}^{\mu \nu} C_{. \mu \nu \omega \nu}^{\lambda}+H_{j i k}^{\lambda} H_{\cdot a \lambda}^{a}-H_{j a}^{\cdot \lambda} H_{\cdot k \lambda}^{a}-(m-2) \Pi_{\mu \nu}^{0} B_{j}^{{ }^{\mu}} B_{k^{\nu}}^{\nu}-g_{j k} I_{\mu \nu}^{0} B^{\mu \nu},
$$

et en multipliant par $g^{j k}$ et en contractant par rapport à $j$ et $k$

$$
g^{a b} R_{a b}=B_{\lambda}^{\omega} B^{\mu \nu} C_{\cdot \mu \nu \omega}^{\lambda}+H_{\cdot a}^{a \cdot \lambda} H_{\cdot b \lambda}^{b}-H_{\cdot a}^{b \cdot \lambda} H_{\cdot b \lambda}^{a}-2(m-1) I_{\mu \nu}^{0} B^{\mu \nu} .
$$

Ces deux équations nous donnent

$$
\begin{aligned}
{ }^{*} I_{j k}^{\dot{j}}= & -\frac{R_{j k}}{m-2}+\frac{g^{a b} R_{a b} g_{j k}}{2(m-1)(m-2)} \\
= & -\frac{1}{m-2} B_{\lambda}^{\omega} B_{j k}^{\mu \nu} C_{. \mu \nu \omega}^{\lambda}+\frac{1}{2(m-1)(m-2)} B_{\lambda}^{\omega} B^{\mu \nu} C_{. \mu \nu \omega}^{\lambda} g_{j k}+I I_{\mu \nu}^{0} B_{j}^{\cdot \mu} B_{k}^{\cdot \nu} \\
& -\frac{1}{m-2}\left(H_{j k}^{\cdot \lambda} H_{\cdot a \lambda}^{a}-H_{j a}^{\cdot \lambda} H_{\cdot k \lambda}^{\alpha}\right)+\frac{1}{2(m-1)(m-2)}\left(H_{\cdot a}^{a \cdot \lambda} H_{\cdot b \lambda}-H_{\cdot a}^{\cdot \cdot \lambda} H_{\cdot b \lambda}^{\alpha}\right) g_{j k},
\end{aligned}
$$

soit,

$$
\begin{aligned}
& \text { (2.3) }{ }^{*} \Pi_{j k}^{\dot{j}}=I_{\mu \nu}^{0} B_{j}^{\mu} B_{k^{\nu}}^{\nu}-\frac{1}{m-2} B_{\lambda}^{\omega} B_{j k}^{\mu \nu} C_{. \mu \nu \omega}^{\lambda}+\frac{1}{2(m-1)(m-2)} B_{\lambda}^{\omega} B^{\mu \nu} C_{. \mu \nu \omega}^{\lambda} g_{j k} \\
& +\frac{1}{m-2} M_{j a}^{\cdot \lambda} M_{\cdot k \lambda}^{a}-\frac{1}{2(m-1)(m-2)} M_{\cdot a}^{b \cdot \lambda} M_{\cdot b \lambda}^{a} g_{j k} \\
& -\frac{1}{m} H_{j k}^{\lambda} H_{\cdot a \lambda}^{a}+\frac{1}{2 m^{2}} H_{\cdot a}^{a \cdot \lambda} H_{\cdot b \lambda}^{b} g_{j k} \text {. }
\end{aligned}
$$

Donc, en tenant compte de (2.1) et de (2.3), on a finalement 


$$
\begin{aligned}
C_{j k}= & { }^{*} \Pi_{j k}^{\dot{0}}-\Pi_{i k}^{\dot{0}} \\
= & -\frac{1}{m-2} B_{\lambda}^{\omega} B_{j k}^{\mu \nu} C_{. \mu \nu \omega}^{\lambda}+\frac{1}{2(m-1)(m-2)} B_{\lambda}^{\omega} B^{\mu \nu} C_{\cdot \mu \nu \omega}^{\lambda} g_{j k} \\
& +\frac{1}{m-2} M_{j a}^{\cdot \lambda} M_{\cdot k \lambda}^{\alpha}-\frac{1}{2(m-1)(m-2)} M_{\cdot a}^{b \cdot \lambda} M_{\cdot b \lambda}^{a} g_{j k},
\end{aligned}
$$

ce qui nous montre bien la propriété conforme du tenseur $C_{j k \text {. }}$

Du tenseur $C_{j k}$, on peut former aussi une scalaire conforme

$$
\begin{aligned}
C=\frac{1}{m} g^{j k} C_{j k} & =\frac{1}{m} g^{j k}\left({ }^{*} \Pi_{j k}^{\dot{0}}-\Pi_{j k}^{\dot{j}}\right) \\
& =-\frac{1}{2 m(m-1)}\left(B_{\lambda}^{\omega} B^{\mu \nu} C_{. \mu \nu \omega}^{\lambda}-M_{\cdot a}^{b \cdot \lambda} M_{\cdot b \lambda}^{\alpha}\right)
\end{aligned}
$$

Cela etant, nous allons, en passant, calculer les valeurs de $\Pi_{P}^{\dot{\theta}}$ En contractant $B_{\lambda P k h}^{i \mu \nu \omega}$ à l'expression de $C_{. \mu \nu \omega,}^{\lambda}$, on obtient

où

$$
B_{\lambda P k h}^{i \mu \nu \omega} C_{\cdot \mu \nu \omega}^{\lambda}=B_{\lambda P k h}^{i \mu \nu \omega} R_{\mu \nu \omega}^{\lambda}+\Pi_{\mu \nu}^{\lambda} B_{P k}^{\mu \nu} \delta_{h}^{i}-\Pi_{\mu \nu}^{0} B_{P h}^{\mu \nu} \delta_{k}^{i}
$$

$$
B_{\lambda P k h}^{i \mu \nu \omega}=B_{\cdot \lambda}^{i} B_{\dot{P}}^{\mu} B_{k}^{\cdot \nu} B_{h}^{\cdot \omega}, \quad B_{P k}^{\mu \nu}=B_{P}{ }^{\mu} B_{k_{k}}^{\nu}
$$

Or, en substituant les équations de Codazzi

$$
B_{\lambda P k h}^{i \mu \nu \omega} R_{\cdot \mu \nu \omega}^{\lambda}=-H_{\cdot k P ; h}^{i}+H_{\cdot h P ; k}^{i}-H_{\cdot k Q}^{i} L_{Q P h}+H_{\cdot h Q}^{i} L_{Q P k}
$$

dans ces équations, on trouve

$$
\begin{aligned}
B_{\lambda P h h}^{i \mu \omega} C_{\mu_{\mu \nu \omega}}^{\lambda}=-H_{{ }_{k P} ; h}^{i}+H_{\cdot h P ; k}^{i}-H_{\cdot{ }_{k Q}}^{i} L_{Q P h}+H_{\cdot h Q}^{i} L_{Q P k} \\
+I_{\mu \nu}^{0} B_{P k}^{\mu \nu} \delta_{h}^{i}-I_{\mu \nu}^{0} B_{P h}^{\mu \nu} \delta_{k}^{i},
\end{aligned}
$$

donc, en posant $i=h$ et en sommant, on obtient

$$
\begin{gathered}
\Pi_{\mu \nu}^{0} B_{P k}^{\mu \nu}=\frac{1}{m-1}\left(B_{\lambda}^{\omega} B_{P k_{k}}^{\mu \nu} C_{\mu \nu \omega}^{\lambda}+H_{\cdot k P ; a}^{a}-H_{\cdot a P ; k}^{a}+H_{\cdot k Q}^{a} L_{Q P a}-H_{\cdot a Q}^{a} L_{Q P k}\right), \\
\text { d'où } \quad \Pi_{\mu \nu}^{0} B_{P h}^{\mu \nu}=\frac{1}{m-1}\left(B_{\lambda}^{\omega} B_{P k}^{\mu \nu} C_{\cdot \mu \nu \omega}^{\lambda}+M_{\cdot k P ; a}^{a}+M_{\cdot k Q}^{a} L_{Q P a}\right) \\
-\frac{1}{m} H_{\cdot a P ; k}^{a}-\frac{1}{m} H_{\cdot a Q}^{a} L_{Q P k},
\end{gathered}
$$

grâce à relation

$$
M_{\cdot k P}^{a}=H_{\cdot k P}^{a}-\frac{1}{m} H_{\cdot b P}^{b} \delta_{k}^{a} .
$$

Donc, en substituant ce resultat dans (1.6), on obtient

$$
I I_{P k}^{\dot{0}}=\frac{1}{m-1}\left(B_{\lambda}^{\omega} B_{P k}^{\mu \nu} C_{\mu \nu \omega}^{\lambda}+M_{\cdot k P ; a}^{a}+M_{\cdot k Q}^{a} L_{Q P a}\right)
$$

§3. Les tenseurs de courbure de $C_{n}$ sont définis par

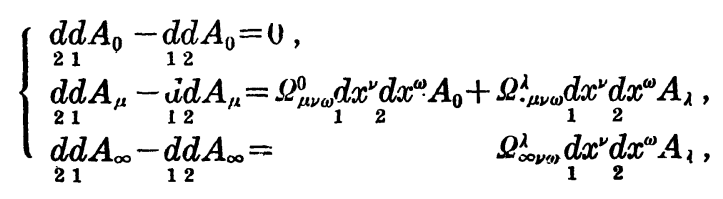


où

$$
\begin{aligned}
& \Omega_{\mu \nu \omega}^{0}=\Pi_{\mu \nu ; \omega}^{0}-\Pi_{\mu \omega ; \nu}^{0}, \quad \Omega_{\infty \nu \omega}^{\lambda}=g^{\lambda \mu} \Omega_{\mu \nu \omega}^{0}, \\
& \Omega_{\cdot \mu \nu \omega}^{\lambda}=R_{\cdot \mu \nu \omega}^{\lambda}+\Pi_{\mu \nu}^{0} \delta_{\omega}^{\lambda}-\Pi_{\mu \omega}^{0} \delta_{\nu}^{\lambda}+g_{\mu \nu} \Pi_{\infty \omega}^{\lambda}-g_{\mu \omega} \Pi_{\infty \nu}^{\lambda},
\end{aligned}
$$

et les tenseurs de courbure du sous-espace $C_{m}$ à connexion conforme induite par

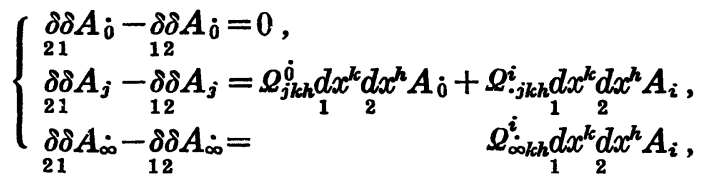

où

$$
\begin{aligned}
& \Omega_{j k h}^{\dot{j}}=\Pi_{j k ; h}^{\dot{0}}-\Pi_{j h ; k}^{\dot{0}}, \quad Q_{\dot{c} k h}^{\dot{i}}=g^{i j} \Omega_{j k h}^{\dot{j}}, \\
& \Omega_{{ }^{j} k h}^{i}=R_{\cdot j k h}^{i}+\Pi_{j k}^{\dot{j}} \delta_{h}^{i}-\Pi_{j h}^{\dot{j}} \delta_{k}^{i}+g_{j k} \Pi_{\dot{\infty} h}^{i}-g_{j h} \Pi_{\dot{\infty} k k}^{i} \text {. }
\end{aligned}
$$

La connexion conforme de l'espace ambiant étant normale, $\boldsymbol{Q}_{\mu \nu \omega}^{0}$ coïncide avec le tenseur de J.M. Thomas et $Q_{\cdot \mu \nu \omega}^{\lambda}$ avec le tenseur conforme de courbure de $\mathrm{H}$. Weyl.

Donc, d'après la notation habituelle, nous les désignerons par $C_{\mu \nu \omega}^{0}$ et $C_{\text {. }{ }_{\mu \nu \omega}}^{\lambda}$ respectivement.

Mais, la connexion induite n'étant pas toujours normale, en substituant

$$
\Pi_{j k}^{\dot{0}}={ }^{*} \Pi_{j k}^{\dot{0}}-C_{j k}
$$

dans (3.5) et (3.6), on obtient respectivement

$$
\begin{aligned}
& \boldsymbol{Q}_{j k h}^{\dot{j}}=C_{j k h}^{\dot{j}}-C_{j k ; k}+C_{j h ; k}, \\
& \boldsymbol{Q}_{\cdot{ }_{j k h}}^{i}=C_{\cdot j k h}^{i}-C_{j k} \delta_{h}^{i}+C_{j h} \delta_{k}^{i}-g_{j k} C_{\cdot h}^{i}+g_{j h} C_{\cdot k}^{i} .
\end{aligned}
$$

où $C_{j k h}^{\dot{0}}$ et $C_{. j k h}^{i}$ sont les tenseurs de courbure de J. M. Thomas et de H. Weyl pour le sous-espace et $C_{. k}^{i}=g^{i j} C_{j k}$.

Or, nous avons

$$
\begin{aligned}
& \text { (3.9) } \underset{21}{d d} d A_{j}-\underset{12}{d d} A_{j}=\left(\Omega_{j k h}^{\dot{0}}+\Pi_{j k P} \Pi_{P h}^{\dot{0}}-\Pi_{j h P} \Pi_{P k}^{\dot{j}}\right) d x_{2}^{k} d x^{h} A_{j} \\
& +\left(\Omega_{\cdot j k h}^{i}+\Pi_{j k P} \Pi_{P h}^{i}-\Pi_{j h P} \Pi_{P k}^{i}\right) d x_{2}^{k} d x^{k} A_{i} \\
& +\left(\Pi_{j k P ; h}-\Pi_{j h P ; k}+\Pi_{j k Q} \Pi_{Q P h}-\Pi_{j h Q} I_{Q P k}\right. \\
& \left.+g_{j k} I_{\dot{\infty} P h}-g_{j h} \Pi_{\dot{\infty} P k}\right) d x^{k} d x^{h} A_{P}, \\
& \text { (3.10) } \underset{21}{d d} A_{P}-\underset{12}{d d} A_{P}=\left(\Pi_{P k ; h}^{\dot{0}}-\Pi_{P h ; k}^{\dot{0}}+\Pi_{P l k}^{a} \Pi_{a h}^{\dot{0}}-\Pi_{P h}^{a} \Pi_{a k}^{\dot{0}}\right. \\
& \left.+I_{P Q k} \Pi_{Q h}^{\dot{0}}-\Pi_{P Q h} \Pi_{Q k}^{\dot{0}}\right) d x_{2}^{k} d x^{h} A_{\dot{0}} \\
& +\left(\Pi_{P k ; h}^{i}-\Pi_{P h ; k}^{i}+\Pi_{Q k}^{i} \Pi_{Q P h}-\Pi_{Q h}^{i} \Pi_{Q P k}\right. \\
& \left.+I_{P k}^{\dot{0}} \delta_{h}^{i}-\Pi_{P h}^{\dot{0}} \delta_{k}^{i}\right) d x_{2}^{k} d x^{h} A_{i} \\
& +\left(I_{P Q k ; h}-I_{P Q h ; k}+\Pi_{P k}^{a} \Pi_{a h Q}-\Pi_{P h}^{a} I I_{a k Q}\right. \\
& \left.+I I_{P R k} I I_{R Q h}-I I_{P R h} \Pi_{R Q k}\right) d x_{2}^{k} d x^{h} A_{Q},
\end{aligned}
$$


et d'autre part

(3.11) $\underset{21}{d d} A_{j}-\underset{12}{d d} A_{j}=\underset{21}{d d}\left(B_{j}^{\cdot \mu} A_{\mu}\right)-\underset{12}{d d}\left(B_{j}^{\cdot \mu} A_{\mu}\right)$

$$
\begin{aligned}
& =\left(B_{j k h}^{\mu \nu \omega} Q_{\mu \nu \omega}^{0}-\frac{1}{m} H_{\cdot a P}^{a} B_{P \lambda} B_{j k h}^{\mu \nu \omega} Q_{\cdot \mu \nu \omega}^{\lambda}\right) d x_{2}^{k} d x^{h} A_{0} \\
& +B_{\lambda j k h}^{i \mu \nu \omega} \Omega_{\cdot \mu \nu \omega}^{\lambda} d x_{1}^{k} d x^{h} A_{i}+B_{P \lambda} B_{j k h}^{\mu \nu \omega} \Omega_{\cdot \mu \nu \omega}^{\lambda} d x_{2}^{k} d x^{h} A_{P},
\end{aligned}
$$

(3.12) $\underset{21}{d d} A_{P}-d d A_{P}=\left(B_{P k h}^{\mu \nu \omega} \Omega_{\mu \nu \omega}^{0}-\frac{1}{m} H_{\cdot a Q}^{a} B_{Q \lambda} B_{P k h}^{\mu \nu \omega} Q_{\cdot \mu \nu \omega}^{\lambda}\right) \underset{1}{d} x_{2}^{k} d x^{h} A_{0}$

$$
+B_{\lambda P k h}^{i \mu \nu \omega} \Omega_{{ }_{\mu \nu \omega \omega}}^{\lambda} d x_{1}^{k} d x^{h} A_{i}+B_{Q \lambda} B_{P k h}^{\mu \nu \omega} \Omega_{\cdot \mu \nu \omega}^{\lambda} d x_{2}^{k} d x^{h} A_{Q} .
$$

Donc, en comparant les coefficients, on obtient, des (3.9) et (3.11),

(3.13) $\quad B_{j k h}^{\mu \nu \omega} \Omega_{\mu \nu \omega}^{0}-\frac{1}{m} H_{\cdot a \lambda}^{a} B_{j k h}^{\mu \nu \omega} \Omega_{\cdot \mu \nu \omega}^{\lambda}=\Omega_{j k h}^{\dot{0}}+\Pi_{j k P} \Pi_{P h}^{\dot{0}}-\Pi_{j h P} \Pi_{P k}^{\dot{0}}$,

(3.14) $B_{\lambda j k h}^{i \mu \nu \omega} \Omega_{. \mu \nu \omega}^{\lambda}=\Omega_{. j k h}^{i}+\Pi_{j k P} \Pi_{P h}^{i}-\Pi_{j h P} \Pi_{P k}^{i}$,

(3.15) $\quad B_{P \lambda} B_{j k h}^{\mu \nu \omega} Q_{\mu \nu \omega}^{\lambda}=\Pi_{j k P ; h}-\Pi_{j h P ; k}+\Pi_{j k Q} \Pi_{Q P h}-\Pi_{j h Q} \Pi_{Q P k}$

et des (3.10) et (3.12)

$$
+g_{j k} \Pi_{\dot{\infty} P h}-g_{j h} \Pi_{\dot{\infty} P k},
$$

(3.16) $\quad B_{P k h}^{\mu \nu \omega} Q_{\mu \nu \omega}^{0}-\frac{1}{m} H_{\cdot a \lambda}^{a} B_{P k h}^{\mu \nu \omega} S_{. \mu \nu \omega)}^{\lambda}=\Pi_{P k ; h}^{\dot{0}}-\Pi_{P h ; k}^{\dot{0}}+\Pi_{P k}^{a} \Pi_{a h}^{\dot{0}}$

$$
-I I_{P h}^{a} \Pi_{a k}^{\dot{0}}+I I_{P Q k} \Pi_{Q h}^{\dot{0}}-\Pi_{P Q h} \Pi_{Q k}^{\dot{0}},
$$

(3.17) $B_{\lambda P k h}^{i \mu \nu \omega} \Omega_{, \mu \nu \omega}^{\lambda}=I I_{P k ; h}^{i}-\Pi_{P h ; k}^{i}+\Pi_{Q k}^{i} \Pi_{Q P h}-\Pi_{Q h}^{i} \Pi_{Q P k}$

$$
+\Pi_{P k}^{\dot{j}} \delta_{h}^{i}-\Pi_{P h}^{\dot{0}} \delta_{j}^{i}
$$

(3.18) $\quad B_{Q \lambda} B_{P k h}^{\mu \nu \omega} \Omega_{, \mu \nu \omega}^{\lambda}=\Pi_{P Q k ; h}-I_{P Q h ; k}+\Pi_{P k}^{a} \Pi_{a h Q}-\Pi_{P h}^{\alpha} \Pi_{a k Q}$

$$
+\Pi_{P R k} \Pi_{R Q h}-\Pi_{P R h} \Pi_{R Q k} \text {. }
$$

Or, si l'on substitue les valeurs (1.6), (2.4), (2.6) et (3.7) dans l'équation (3.13), on obtient

(3.19) $\quad B_{j k h}^{\mu \nu \omega} C_{\mu \nu \omega \omega}^{0}-\frac{1}{m} H_{\cdot a \lambda}^{a} B_{j k h}^{\mu \nu \omega} C_{. \mu \nu \omega}^{\lambda}$

$$
\begin{aligned}
- & {\left[\frac{1}{m-2} B_{\lambda}^{\omega} B_{j k}^{\mu \nu} C_{\cdot \mu \nu \omega}^{\lambda}-\frac{1}{2(m-1)(m-2)} B_{\lambda}^{\omega} B^{\mu \nu} C_{. \mu \nu \omega}^{\lambda} g_{j k}\right]_{; h} } \\
+ & {\left[\frac{1}{m-2} B_{\lambda}^{\omega} B_{j h}^{\mu \nu} C_{\cdot \mu \nu \omega}^{\lambda}-\frac{1}{2(m-1)(m-2)} B_{\lambda}^{\omega} B^{\mu \nu} C_{\cdot \mu \nu \omega}^{\lambda} g_{j h}\right]_{; k} } \\
- & \frac{1}{m-1} M_{j k P} B_{\lambda}^{\omega} B_{P h}^{\mu \nu} C_{\cdot \mu \nu \omega}^{\lambda}+\frac{1}{m-1} M_{j h P} B_{\lambda}^{\omega} B_{P k}^{\mu \nu} C_{\cdot \mu \nu \omega}^{\lambda} \\
=C_{j k h}^{\dot{j}} & -\left[\frac{1}{m-2} M_{j a}^{\cdot{ }^{\lambda}} M_{\cdot k \lambda}^{a}-\frac{1}{2(m-1)(m-2)} M_{\cdot a}^{b \cdot \lambda} M_{\cdot b \lambda}^{a} g_{j k}\right]_{; h} \\
+ & {\left[\frac{1}{m-2} M_{j a}^{\cdot \cdot \lambda} M_{\cdot h \lambda}^{\alpha}-\frac{1}{2(m-1)(m-2)} M_{\cdot a}^{b \cdot \lambda} M_{\cdot b \lambda}^{a} g_{j h}\right]_{; k} }
\end{aligned}
$$




$$
\begin{aligned}
& +\frac{1}{m-1} M_{j k P}\left(M_{\cdot h P ; a}^{a}+M_{\cdot h Q}^{a} L_{Q P a}\right) \\
& -\frac{1}{m-1} M_{j h P}\left(M_{\cdot k P ; a}^{a}+M_{\cdot k Q}^{a} L_{Q P a}\right) .
\end{aligned}
$$

Dans le cas de lespace conforme à un espace euclidien, c'est-à-dire, dans le cas où les tenseurs $C_{\mu \nu \omega}^{0}$ et $C_{. \mu \nu \omega}^{\lambda}$ s'annulent tous les deux, nous avons déjà rencontré ces équations ${ }^{1}$.

Cela étant, substituons cette fois (1.6), (2.4) et (3.8) dans (3.14), alors, on aura

$$
\begin{aligned}
& B_{\langle j k h}^{i \mu \omega \omega} C_{\cdot \mu \nu \omega}^{\lambda}-\left[\frac{1}{m-2} B_{\lambda}^{\omega} B_{j k}^{\mu \nu} C_{. \mu \nu \omega}^{\lambda}-\frac{1}{2(m-1)(m-2)} B_{\lambda}^{\omega} B^{\mu \nu} C_{. \mu \nu \omega}^{\lambda} g_{j k}\right] \partial_{h}^{i} \\
& +\left[\frac{1}{m-2} B_{\lambda}^{\omega} B_{j h}^{\mu \nu} C_{. \mu \nu \omega}^{\lambda}-\frac{1}{2(m-1)(m-2)} B_{\lambda}^{\omega} B^{\mu \nu} C_{\mu \nu \omega \omega}^{\lambda} g_{j h}\right] \delta_{k}^{i} \\
& -g_{j k}\left[\frac{1}{m-2} B_{\lambda}^{\omega} B_{a h}^{\mu \nu} C_{. \mu \nu \omega}^{\lambda}-\frac{1}{2(m-1)(m-2)} B_{\lambda}^{\omega} B^{\mu \nu} C_{. \mu \nu \omega}^{\lambda} g_{a h}\right] g^{a i} \\
& +g_{j h}\left[\frac{1}{m-2} B_{\lambda}^{\omega} B_{a k}^{\mu \nu} C_{\cdot \mu \nu \omega}^{\nu}-\frac{1}{2(m-1)(m-2)} B_{\lambda}^{\omega} B^{\mu \nu} C_{\cdot \mu \nu \omega}^{\lambda} g_{a k}\right] g^{a i} \\
& =C_{\cdot j k h}^{i}-M_{j k^{\lambda}} M_{\cdot h \lambda}^{i}+M_{\ddot{j h}^{\lambda}} M_{\cdot k \lambda}^{i} \\
& -\left[\frac{1}{m-2} M_{j a}^{\cdot \lambda} M_{\cdot k \lambda}^{a}-\frac{1}{2(m-1)(m-2)} M_{\cdot a}^{b \cdot \lambda} M_{\cdot b \lambda}^{a} g_{j k}\right] \delta_{h}^{i} \\
& +\left[\frac{1}{m-2} M_{j a}^{\cdot \lambda} \cdot M_{\cdot h \lambda}^{a}-\frac{1}{2(m-1)(m-2)} M_{\cdot a}^{b \cdot \lambda} M_{\cdot b \lambda}^{a} g_{j h}\right]_{\delta_{k}^{i}} \\
& -g_{j k}\left[\frac{1}{m-2} M_{\cdot a}^{i \cdot \lambda} M_{\cdot h \lambda}^{a}-\frac{1}{2(m-1)(m-2)} M_{\cdot a}^{b \cdot \lambda} M_{\cdot b \lambda}^{a} \delta_{h}^{i}\right] \\
& +g_{j h}\left[\frac{1}{m-2} M_{\cdot a}^{i \cdot \lambda} M_{\cdot k \lambda}^{a}-\frac{1}{2(m-1)(m-2)} M_{\cdot a}^{b \cdot \lambda} M_{\cdot b \lambda}^{a} \delta_{k}^{i}\right] \text {. }
\end{aligned}
$$

Ce sont les équations conformes de Gauss que nous avons déjà trouvées d'une autre manière ${ }^{2}$. Substituons cette fois (1.6) et

$$
I I_{\dot{\infty} P k}=I_{P k}^{\dot{0}}=\frac{1}{m-1}\left(B_{\lambda}^{\omega} B_{P k}^{\mu \nu} C_{\cdot \mu \nu \omega}^{\lambda}+M_{\cdot k P ; a}^{a}+M_{\cdot k Q}^{a} L_{Q P a}\right)
$$

dans (3.15), alors on aura

$$
\begin{aligned}
& B_{\Gamma \lambda} B_{j k h}^{\mu \nu \omega} C_{\cdot \mu \nu \omega}^{\lambda}-\frac{1}{m-1} g_{j k} B_{\lambda}^{\omega} B_{P h}^{\mu \nu} C_{\mu \nu \omega}^{\lambda}+\frac{1}{m-1} g_{j h} B_{\lambda}^{\omega} B_{P k}^{\mu \nu} C_{\mu_{\mu \nu \omega}}^{\lambda} \\
&= M_{j k P ; h}-M_{j h P ; k}+M_{j k Q} L_{Q P h}-M_{j h Q} L_{Q P k} \\
&+\frac{1}{m-1} g_{j k}\left(M_{\cdot h P ; a}^{a}+M_{\cdot h Q}^{a} L_{Q P a}\right) \\
&-\frac{1}{m-1} g_{j h}\left(M_{\cdot k P ; a}^{a}+M_{\cdot k Q}^{a} L_{Q P a}\right)
\end{aligned}
$$

1) K. Yano et Y. Mutô: Sur le théorème fondamental..., déjà cité, (48).

2) K. Yano: Sur les équations de Gauss....., déjà cité, (3.14). 
Ce sont les équations conformes de Codazzi ${ }^{1)}$

Cela étant, substituons (1.6), (2.4) et (2.6) dans (3.16), alors on trouve

$$
\begin{aligned}
& B_{P k h}^{\mu \nu \omega} C_{\mu \nu \omega}^{0}-\frac{1}{m} H_{\cdot a \lambda}^{a} B_{P k h}^{\mu \nu \omega} C_{\mu \nu \omega}^{\lambda} \\
& -\frac{1}{m-1}\left(B_{\lambda}^{\omega} B_{P k}^{\mu \nu} C_{. \mu \omega \omega}^{\lambda}\right)_{h}+\frac{1}{m-1}\left(B_{\nu}^{\omega} B_{P h}^{\mu \nu} C_{\mu \nu \omega \omega}^{\lambda}\right)_{; k} \\
& -\frac{1}{m-2} M_{\cdot k P}^{\alpha} B_{\lambda}^{\alpha} B_{a h}^{\mu \nu} C_{\mu \nu \omega a r}^{\lambda}+\frac{1}{m-2} M_{\cdot k P}^{\alpha} B_{\lambda}^{\omega} B_{a k}^{\mu \nu} C_{. \mu \nu \omega}^{\lambda} \\
& -\frac{1}{m-1} L_{P Q k} B_{\lambda}^{\omega} B_{Q h}^{\mu \nu} C_{\mu \nu \omega}^{\lambda}+\frac{1}{m-1} L_{P Q h} B_{\lambda}^{\omega} B_{Q k}^{\mu \nu} C_{. \mu \nu \omega}^{\lambda} \\
& =\frac{1}{m-1}\left(M_{\cdot k P ; a}^{a}+M_{\cdot k Q}^{a} L_{Q P a}\right)_{; h} \\
& -\frac{1}{m-1}\left(M_{\cdot h P ; a}^{a}+M_{\cdot h Q}^{a} L_{Q P a}\right)_{; k} \\
& +\frac{1}{m-2} M_{\cdot k P}^{a} R_{a h}-\frac{1}{m-2} M_{\cdot h P}^{a} R_{a k} \\
& -\frac{1}{m-2} M_{\cdot k P}^{a} M_{\ddot{a} b^{\lambda}} M_{\cdot h \lambda}^{b}+\frac{1}{m-2} M_{\cdot h P}^{a} M_{a b}^{\lambda} M_{\cdot k \lambda}^{b} \\
& +\frac{1}{m-1} L_{P Q k}\left(M_{\cdot \cdot h Q ; a}^{a}+M_{\cdot h R}^{a} L_{R Q a}\right) \\
& -\frac{1}{m-1} L_{P Q h}\left(M_{\cdot k Q ; a}^{a}+M_{\cdot k R}^{a} L_{R Q a}\right) \text {. }
\end{aligned}
$$

Nous avons aussi rencontré ces équations dans le cas de l'espace conforme à l'espace euclidien".

Les équations (3.17) ne donnent pas les nouvelles équations, ce sont les équations conformes de Codazzi.

En substituant finalement (1.6) dans (3.18), nous avons

$$
\begin{aligned}
B_{Q \lambda} B_{P k h}^{\mu \nu \omega} C_{. \mu \nu \omega}^{\lambda}=L_{P Q k ; h}-L_{P Q h ; k}-M_{\cdot k P}^{a} M_{a h Q}+M_{. h P}^{a} M_{a k Q} \\
+L_{P R k} L_{R Q h}+L_{P R h} L_{R Q k},
\end{aligned}
$$

ce sont les équations conformes de Ricci.

1) K. Yano: Sur les équations de Codazzi ..., déjà cité, (25).

2) K. Yano et $Y$. Mutô: Sur le théorème fondamental..., déjà cité, (49). 